\title{
Timing and cross-talk properties of BURLE multi-channel MCP PMTs
}

\author{
Samo Korpar* \\ Faculty of Chemistry and Chemical Engineering, University of Maribor, \\ and Jožef Stefan Institute, Ljubljana, Slovenia \\ E-mail: 'samo.korparâjo

\section{Peter Križan} \\ Faculty of Mathematics and Physics, University of Ljubljana, \\ and Jožef Stefan Institute, Ljubljana, Slovenia

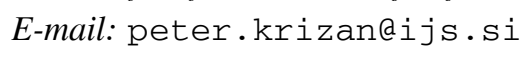

\section{Rok Pestotnik} \\ Jožef Stefan Institute, Ljubljana, Slovenia \\ E-mail: rrok.pestotnik@ijo.sí
}

\begin{abstract}
We report on studies of the two types of BURLE multi-anode micro-channel plate (MCP) PMTs; one with 64 channels and $25 \mu \mathrm{m}$ pores and the other with 4 channels and $10 \mu \mathrm{m}$ pores. Possible applications of these tubes are for RICH detectors and time-of-flight counters. We have investigated their timing properties and cross-talk. The origin of the cross talk between the channels was investigated by studying the correlations between signals on neighbouring pads, their amplitudes and time of arrival. We have also studied the influence of cross-talk on timing and spatial resolution.
\end{abstract}

International workshop on new photon-detectors PD07

June 27-29 2007

Kobe University, Kobe, Japan

\footnotetext{
* Speaker.
} 


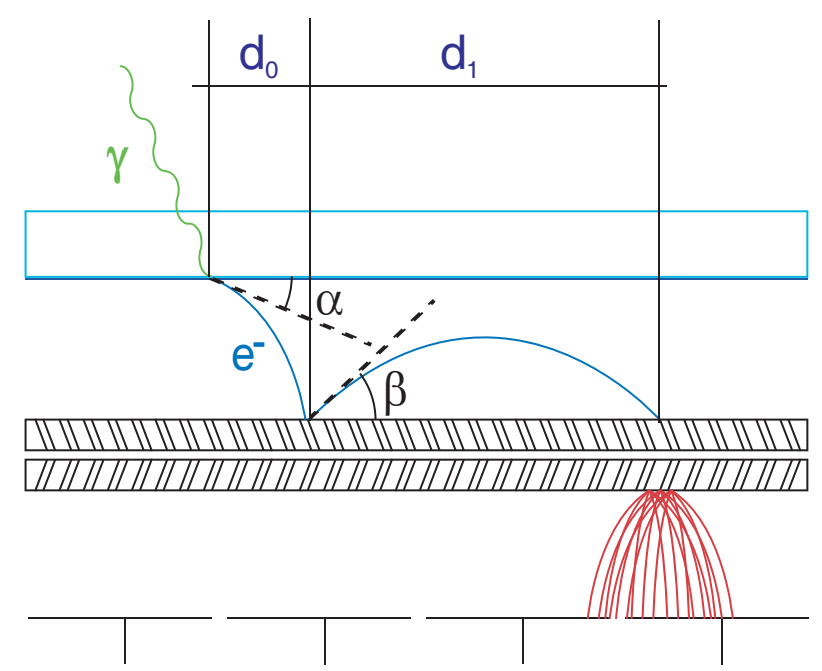

Figure 1: Physical processes that lead to a signal on the anode of a MCP-PMT. The distance between photocathode and MCP is around $6 \mathrm{~mm}$ and potential difference applied to them during the tests was $200 \mathrm{~V}$.

\section{Introduction}

For the upgrade of particle identification systems at B-factories three different types of Cherenkov detectors are proposed: a Time-Of-Propagation (TOP) counter, a proximity focusing aerogel RICH, both at the Belle experiment [i] $\operatorname{li}_{1}$ and a focusing DIRC as an upgrade of BaBar DIRC [iㄹㄱ. All these counters require photon detectors that work in high magnetic fields of about $1.5 \mathrm{~T}$, have pad size of few $\mathrm{mm}$ and timing resolution around $50 \mathrm{ps}$.

A most promising candidate for photon detector is a multi channel plate photomultiplier (MCP-PMT). In this device the dynode structure is replaced with MCP amplification. One of the photon detector candidates is the 64 channel Burle 85011 MCP-PMT tube. Two types of the tube are being tested, one with $25 \mu \mathrm{m}$ pores, and the other with $10 \mu \mathrm{m}$ pores. While we have already

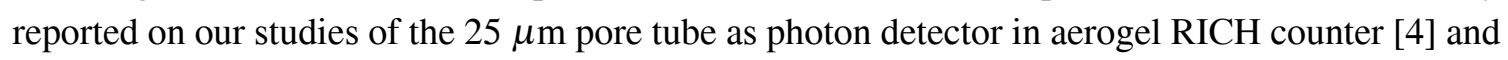
its ability to serve also for TOF [S్̄'], we discuss in the present contribution our measurements of timing and crosstalk properties of $25 \mu \mathrm{m}$ and $10 \mu \mathrm{m}$ pore versions.

In order to better understand the parameters that determine the position resolution and the timing resolution, we show in Fig.'I' of a MCP-PMT. Different angles $\alpha$ at which the photoelectron exits the photocathode, as well as backscattering of the photoelectron on the microchannel plate, contribute to the broadening of both position and timing distributions.

\section{Experimental Set-up}

As a photon source we used a $406 \mathrm{~nm}$ picosecond PiLas laser. The laser light was first attenuated by neutral density filters and guided into a light tight box along the optical fibre, with the far end attached to a computer controlled 2D stage. The light was focused by a biconvex lens onto the photochatode of a MCP-PMT (Fig.루) 

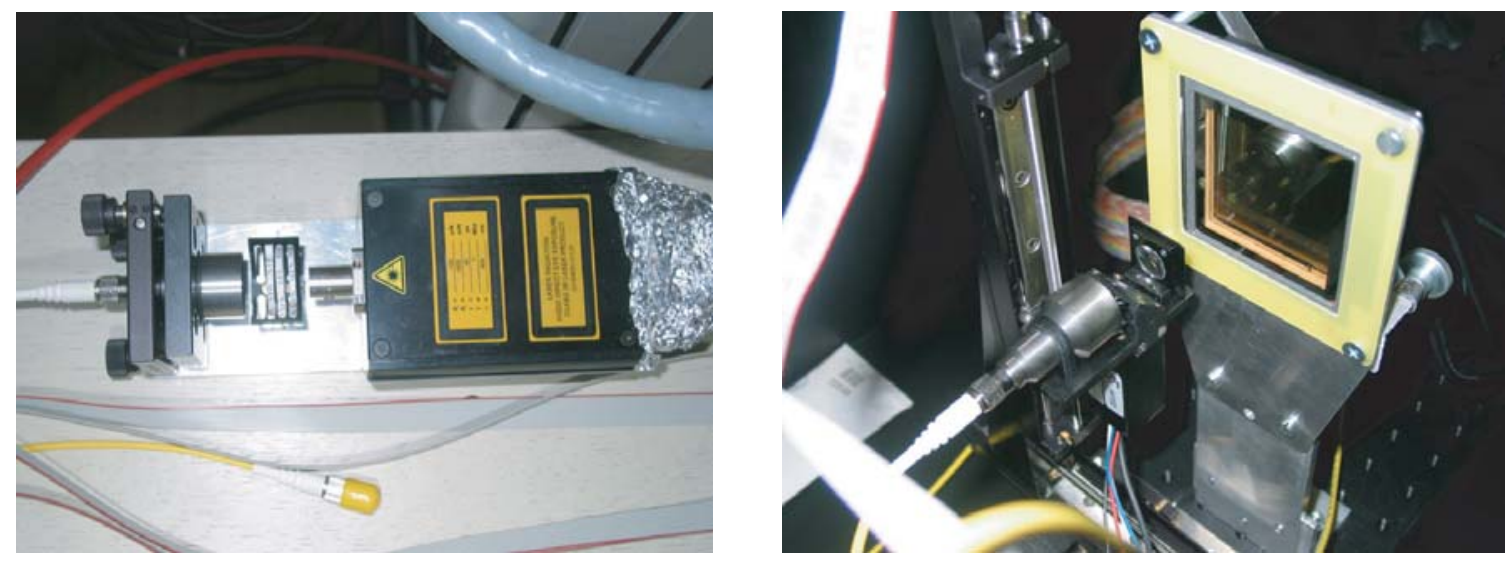

Figure 2: Apparatus for the measurement of single photons with the MCP-PMT. Left photo: Laser unit with filter and light guide. Right photo: Exit end of light guide with focusing lens and MCP-PMT detector in dark box.

The anode signal was amplified by an Ortec FTA820 amplifier. After amplification, the signal was split in two branches; one for the timing and the other for the signal amplitude measurement. The timing signal was discriminated by a Phillips 708 module (NIM). The time of the pulse was measured by the Kaizu Works KC3781A TDC (CAMAC) with 25 ps per channel. Pulse hight was registered in a CAEN V965 charge sensitive ADC (VME). The start signal was determined by the laser control unit.

\section{Results}

From a plot of the pulse height versus pulse time, we have obtained corrections for the pulse arrival time. After applying these corrections, the timing distribution has a main peak with rms of

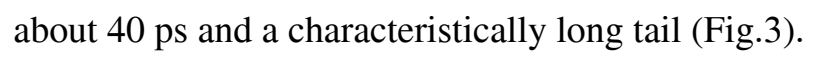

The position dependence of the time distributions of signals from both, the 4-channel and the 64-channel MCP-PMT are shown in Fig.jint. It is seen that signals at pad boundaries and especially at the edges of the sensitive surface tend to be somewhat delayed.

The origin of the cross talk between the channels was investigated by studying the correlations between signals on neighbouring pads, their amplitudes and time of arrival. Fig. ${ }_{-1}^{5} \overline{1}_{1}$ shows the position dependence of the fraction of the total signal that appears on one anode pad for the 4-channel MCP-PMT. In the boundary region that is about $3 \mathrm{~mm}$ wide charge sharing can be used to improve the position resolution. The distribution on the right hand side of Fig.5.5. shows the position distribution of the number of events for which the pulse is equally divided between the two neighbouring pads. The width of this distribution mainly comes from the spread of photoelectron impact point on the MCP due to its initial energy $(\approx 1 \mathrm{eV})$. It can be reduced by smaller distance between the photocathode and MCP and higher potential difference between them.

Fig. of MCP-PMT ( 4 channel and 64 channel). The contributions of cross talk are seen to be relatively small but to extend about $12 \mathrm{~mm}$ beyond the pad boundary in accordance with the estimated maximum range of photoelectrons backscattered on the multichannel plate. 

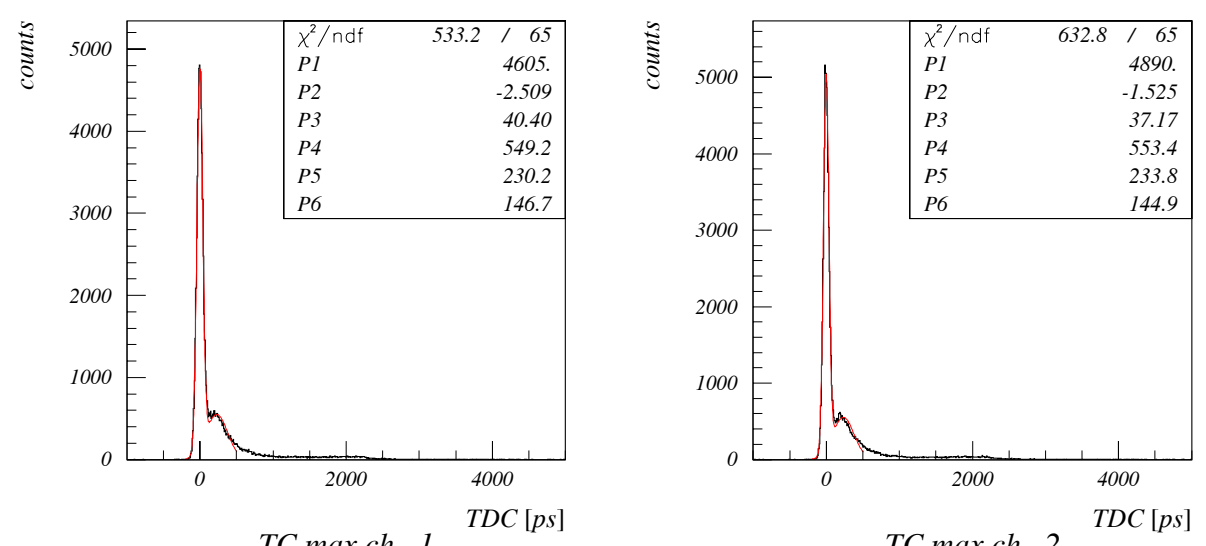

TC max ch. 1
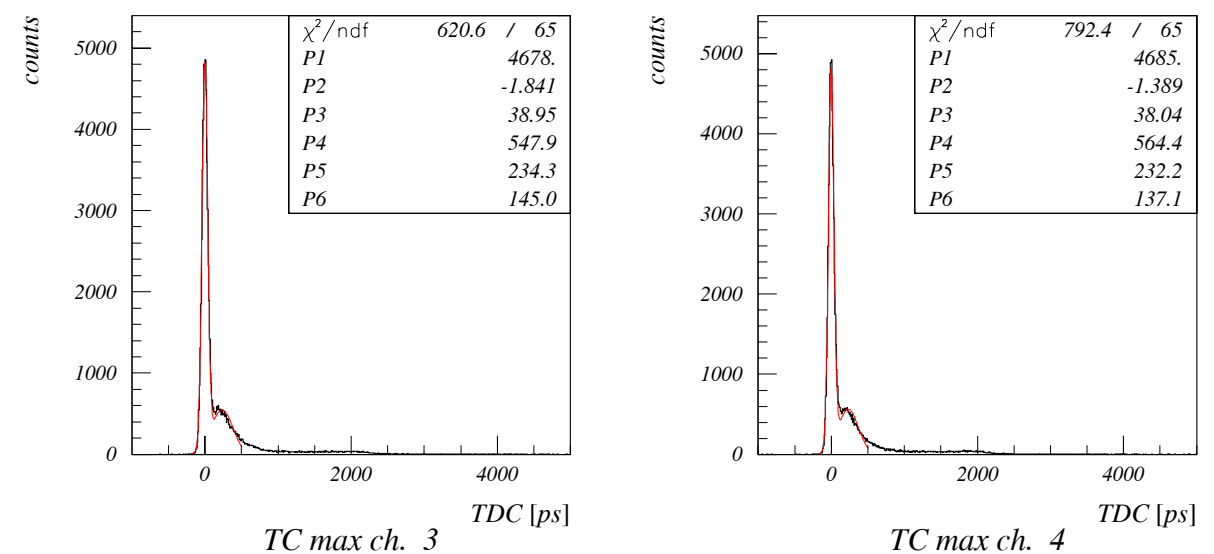

Figure 3: Corrected TDC distributions of all four channels.
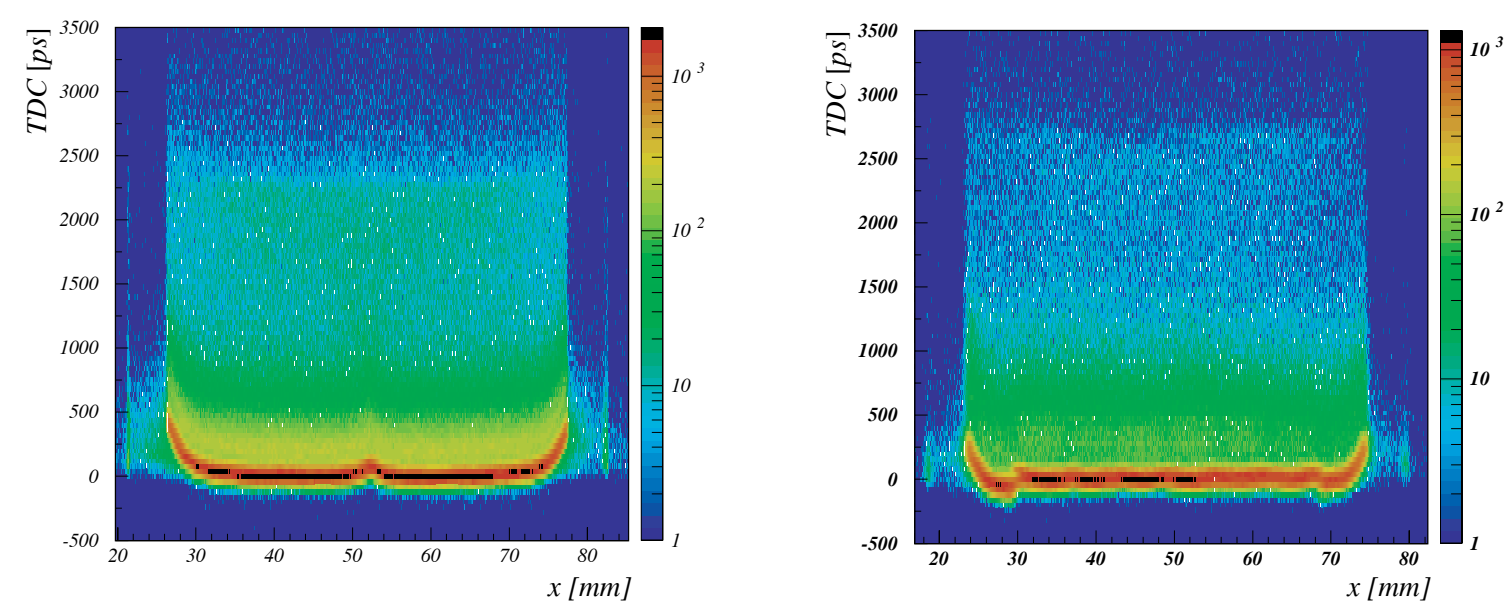

Figure 4: Position (x) dependence of the time distribution of single photon signals for a 2 x 2 channel MCP-PMT (left) and for an 8 x 8 channel MCP-PMT (right). 

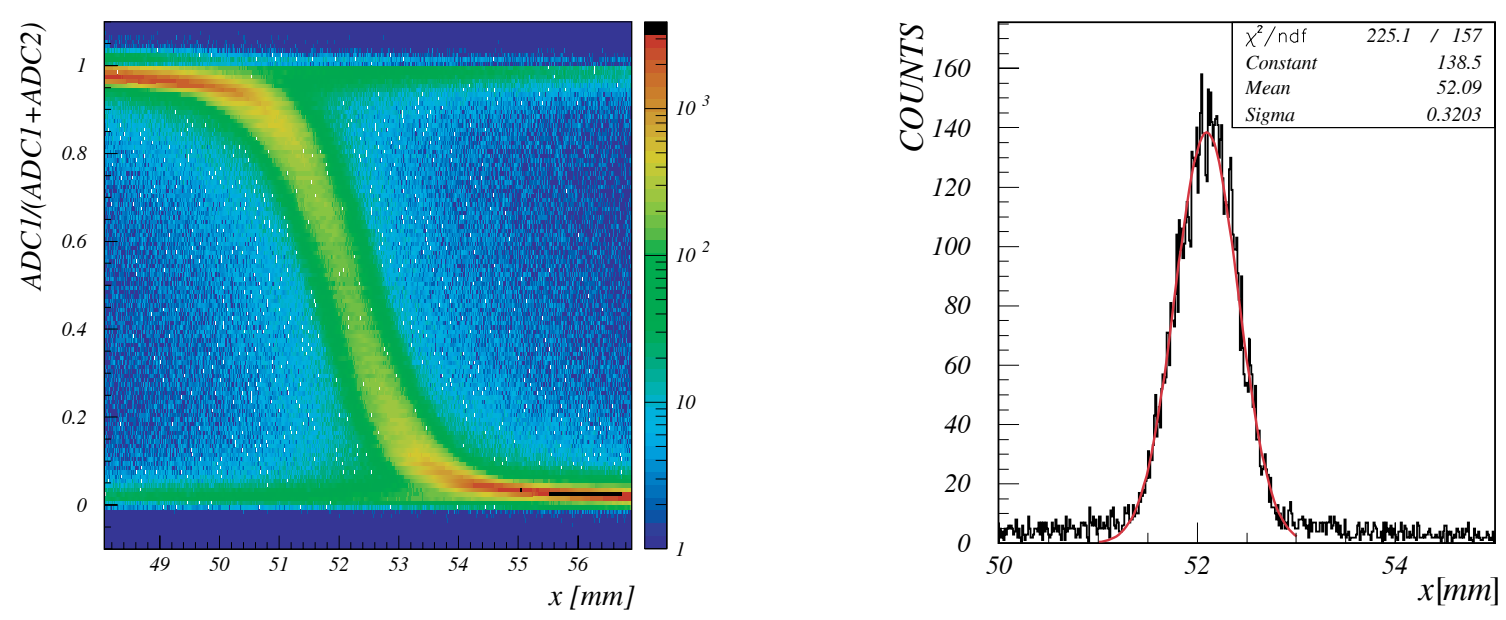

Figure 5: Position (x) dependence of the signal fraction appearing on the left pad with the boundary between the pads seen at $x=52 \mathrm{~mm}$ (left figure). Distribution of events for which the pulse is equally distributed between the two neighbouring pads (right figure).
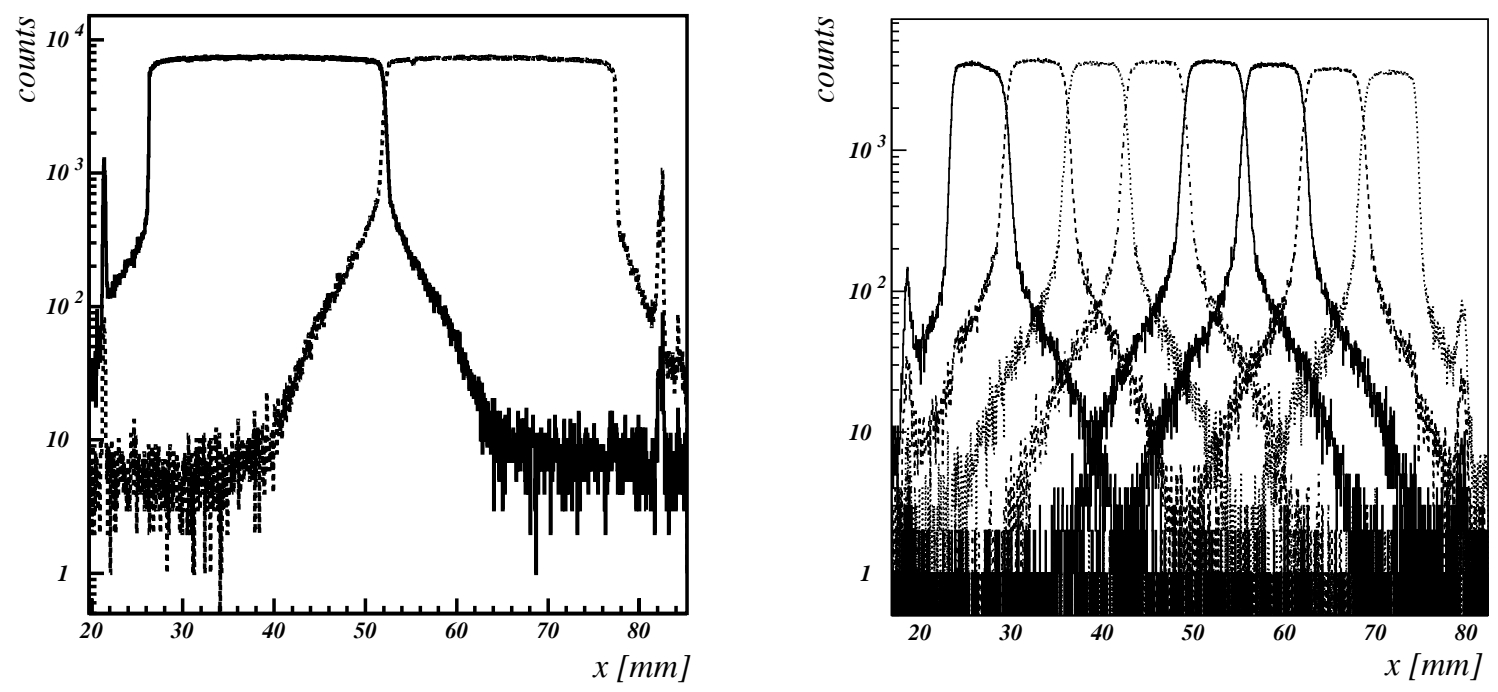

Figure 6: Individual pad count rates as a function of position (x) across 2 channels of the 4 channel MCP-PMT (left) and across 8 channels of the 64 channel MCP-PMT (right).

\section{References}

[1] K. Abe et al. (edited by S. Hashimoto, M. Hazumi, J. Haba, J. W. Flanagan and Y. Ohnishi), Letter of Intent for KEK Super B Factory, KEK report 2004-04, http://belle.kek.jp/superb/

[2] C. Field et al., Nucl. Instrum. Meth. A 553 (2005) 96.

[3] BURLE 85011 data sheet: http//www.burle.com/cgi-bin/byteserver.pl/pdf/85011-501.pdf

[4] S. Korpar et al., Proceedings of the IEEE Nuclear Science Symposium, Rome, Italy, October 17-22, 2004; P. Krizan et al., Nucl. Instrum. Meth. A 567 (2006) 124.

[5] P. Krizan et al., Nuclear Physics B (Proc. Suppl.) 172 (2007) 227. 